\title{
De novo design of organic photocatalysts: bithiophene derivatives for the visible-light induced $\mathrm{C}$-H functionalization of heteroarenes
}

\author{
Cecilia Bottecchia ${ }^{\mathrm{a} \dagger}$, Raúl Martín ${ }^{\mathrm{b} \dagger}$, Irini Abdiaj ${ }^{\mathrm{c}}$, Ettore Crovini ${ }^{\mathrm{a}}$, Jesús Alcazarc ${ }^{\mathrm{c}}$, Jesús \\ Orduna $^{\mathrm{d}}$, María Jesús Blesa ${ }^{\mathrm{d}}$, José R. Carrillo $^{\mathrm{b} *}$, Pilar Prieto ${ }^{\mathrm{b}}$ and Timothy Noël ${ }^{\mathrm{a} *}$
}

a Department of Chemical Engineering and Chemistry, Micro Flow Chemistry and Synthetic Methodology, Eindhoven University of Technology, Den Dolech 2, 5612 AZ Eindhoven, The Netherlands. E-Mail: t.noel@tue.nl

b Departamento de Química Orgánica, Facultad de Ciencias y Tecnologías Químicas, Universidad de Castilla-La Mancha-IRICA, 13071 Ciudad Real, Spain. Email: joseramon.carrillo@uclm.es

c Janssen Research \& Development, Calle Jarama 75A, 45007 Toledo, Spain.

d Departamento de Química Orgánica, Facultad de Ciencias-Instituto de Ciencias de Materiales de Aragón, Universidad de Zaragoza-CSIC, 50009-Zaragoza, Spain.

$\dagger$ These authors contributed equally to this work.

Received: ((will be filled in by the editorial staff))

Supporting information for this article is available on the WWW under http://dx.doi.org/10.1002/adsc.201\#\#\#\#\#\#.((Please delete if not appropriate))

\begin{abstract}
Herein, we report the de novo synthesis and characterization of a series of substituted bithiophene derivatives as novel and inexpensive organic photocatalysts.

DFT calculations were used to predict a priori their absorption spectra and redox potentials, which were then confirmed with empirical data. The photocatalytic activity of this novel class of organic photoredox catalyst was demonstrated in two visible-light mediated strategies for the $\mathrm{C}-\mathrm{H}$ functionalization of heteroarenes. The implementation of these strategies in a continuous-flow photo-microreactor afforded moderate to excellent yields within few minutes of reaction time. Due to their straightforward synthesis, low cost and good photocatalytic properties we believe that the proposed bithiophene derivatives could be employed as a new class of organic photoredox catalysts
\end{abstract}

Keywords: Organic photocatalysts, Photoredox catalysis, DFT calculations, C-H functionalization, Continuous flow

Visible-light photoredox catalysis received increasing attention in the last decade as a powerful strategy enabling novel transformations under mild reaction conditions (i.e. room temperature, atmospheric pressure and visible-light irradiation). ${ }^{[1]}$ In photoredox catalysis, the ability of a photocatalyst to absorb light in the visible light range, reach an excited state and then undergo either energy or electron transfers with the substrates of interest is paramount. ${ }^{[2]}$ Generally speaking, Ir and $\mathrm{Ru}$ polypyridyl complexes are among the most employed photocatalyst, offering excellent redox potentials in their excited states and long-lived exciton lifetimes. ${ }^{[3]}$ However, the main drawbacks associated with their use are their prohibitive costs and scarce availability. ${ }^{[4]}$
More recently, a number of studies demonstrated that organic photocatalysts often provide valuable and inexpensive alternatives to transition metals. ${ }^{[4-5]}$ Nevertheless, the choice of a suitable organic photocatalyst might be limited for reactions proceeding in acidic medium (e.g. Eosin $\mathrm{Y}$ is $\mathrm{pH}$ sensitive) or in reducing environments promoting photobleaching. ${ }^{[6]}$

In this light, the development of novel classes of organic photoredox catalysts with good redox properties, and largely available or easily prepared in high yields would contribute to further advance research in photoredox catalysis. ${ }^{[7]}$

Due to their functional properties and versatility deriving from the ease of functionalization of the ring as well as the lengthening of the chain, thiophene based materials are of broad interest across multiple disciplines. For example, in organic electronics their semiconductor properties are exploited in organic field-effect transistors (OFETs) and in photovoltaic devices. ${ }^{[8]}$ However, examples showing their use in the field of photoredox catalysis remain scarce. ${ }^{[9]}$

Thus, we became interested in probing the photocatalytic properties of a series of 2,2' bithiophene-based derivatives decorated with different electron donating and electron withdrawing groups (see Scheme S1 in SI).

In order to rapidly evaluate the suitability of said structures as photoredox catalysts, we reasoned that computational studies might represent a useful tool to predict their properties a priori. Specifically, through TD-DFT ${ }^{[10]}$ calculations (M06-2X ${ }^{[11]}$, using CPCM ${ }^{[12]}$ see SI for further details), the absorption maximum and emission maximum of this class of compounds were calculated, together with their redox potentials. Based on this theoretical information and taking into 
account the commercial availability of the required starting materials, we selected a cluster of six molecules to be synthesized (Figure 1). Photocatalysts $\mathbf{A}$ to $\mathbf{F}$ were all obtained in good to excellent yields through a one-step double Sonogashira cross-coupling, starting from inexpensive and readily available alkynyl precursors (see experimental section and general procedure 1 in SI). ${ }^{[13]}$ The optimized procedure was performed with minimal amount of solvent, under microwave irradiation and employing recycled Pd catalyst.

Gratifyingly, the calculated theoretical values for the maximum absorption were found to be in good agreement with the experimental measurements (Figure 1). The UV-Visible spectra of all of the studied compounds showed a unique absorption band in the $400 \mathrm{~nm}$ region with significant tailing towards the blue light region, thus supporting our initial hypothesis that these compounds might be useful in visible-light driven catalytic reactions.

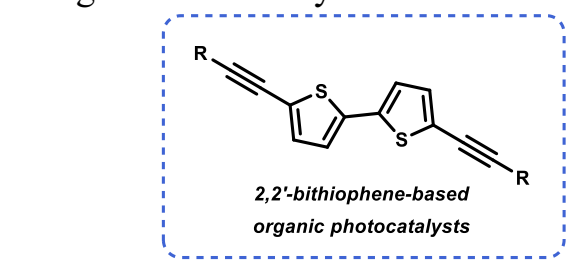

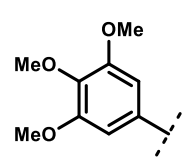

A:<smiles>CI(C)c1ccccc1C(F)(F)F</smiles>

D:<smiles>COc1cc(OC)cc(I(C)C)c1</smiles>

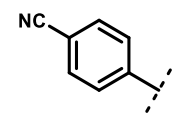

E:

\begin{tabular}{lcccccc}
\hline & A & B & C & D & E & F \\
\hline Calc. Abs $_{\max }$ & $401 \mathrm{~nm}$ & $399 \mathrm{~nm}$ & $407 \mathrm{~nm}$ & $401 \mathrm{~nm}$ & $410 \mathrm{~nm}$ & $417 \mathrm{~nm}$ \\
Exp. Abs $_{\max }$ & $393 \mathrm{~nm}$ & $388 \mathrm{~nm}$ & $390 \mathrm{~nm}$ & $390 \mathrm{~nm}$ & $398 \mathrm{~nm}$ & $400 \mathrm{~nm}$ \\
\hline Calc. $\mathbf{E}_{1 / 2} \mathbf{0 x}$ & $1.25 \mathrm{~V}$ & $1.26 \mathrm{~V}$ & $1.03 \mathrm{~V}$ & $1.28 \mathrm{~V}$ & $1.51 \mathrm{~V}$ & $1.26 \mathrm{~V}$ \\
\hline Exp. $\mathbf{E}_{1 / 2} \mathbf{0 x}$ & $1.08 \mathrm{~V}$ & $1.22 \mathrm{~V}$ & $1.02 \mathrm{~V}$ & $1.34 \mathrm{~V}$ & $1.34 \mathrm{~V}$ & $1.33 \mathrm{~V}$ \\
\hline Calc. $\mathbf{E}_{1 / 2} \mathrm{red}$ & $-2.22 \mathrm{~V}$ & $-2.18 \mathrm{~V}$ & $-2.24 \mathrm{~V}$ & $-2.0 \mathrm{~V}$ & $-1.90 \mathrm{~V}$ & $-2.05 \mathrm{~V}$ \\
\hline${\text { Calc } \mathbf{E}_{1 / 2}^{*} \mathbf{r e d}}$ & $0.53 \mathrm{~V}$ & $0.57 \mathrm{~V}$ & $0.68 \mathrm{~V}$ & $0.74 \mathrm{~V}$ & $0.78 \mathrm{~V}$ & $0.58 \mathrm{~V}$ \\
${\text { Calc } \mathbf{E}_{1 / 2}^{*} \mathbf{0 x}}$ & $-1.49 \mathrm{~V}$ & $-1.49 \mathrm{~V}$ & $-1.38 \mathrm{~V}$ & $-1.46 \mathrm{~V}$ & $-1.17 \mathrm{~V}$ & $-1.37 \mathrm{~V}$
\end{tabular}

Figure 1: Overview of the six 2,2'-bisthiophene-based photocatalyst synthesized and comparison of their calculated vs experimental properties. All potential values are reported vs SCE (Saturated Calomel Electrode). For details on the calculations and on the experimental data we refer to SI. Calc $=$ calculated values. Exp $=$ experimental values.

The redox properties of the reported bithiophenes were followed by Cyclic Voltammetry (CV) and Differential Pulse Voltammetry (DPV) in $\mathrm{CHCl}_{3}$ (for the voltammograms see SI section 3) and matched closely the calculated theoretical values (Figure 1).

As expected, compounds $\mathbf{A}, \mathbf{B}$ and $\mathbf{C}$, bearing electron-donating groups, showed lower oxidation potential than compounds $\mathbf{D}$ and $\mathbf{E}$, bearing electron- withdrawing groups. Moreover, a good correlation between the experimental oxidation potentials and the Hammett constant values for the substituents on the arene ring was found (see SI section 5). Notably, such correlation hints at the possibility to carefully design other members of this photocatalytic series with increasing redox potential by further modulating the nature and position of the substituents on the aryl ring. For all members of the series, the theoretical reduction potentials were calculated to be higher than $-2 \mathrm{~V}$, and thus could not be confirmed experimentally due to the limited potential window of $\mathrm{CHCl}_{3 .}{ }^{[14]} \mathrm{By}$ combining the calculated oxidation/reduction potentials with the $\mathrm{E}_{0,0}$ values, the excited state potentials for compounds $\mathbf{A}$ to $\mathbf{F}$ were also derived (Figure 2, and SI section 2). Notably, all compounds of the series showed excited state potentials comparable to commonly used photocatalysts. ${ }^{[3-4]}$

To test the activity of the bithiophene derivatives as photoredox catalysts, we commenced our investigations by employing them in the $\mathrm{C}-\mathrm{H}$ functionalization of heteroarenes with malonate derivatives. $^{[15]}$

Table 1: Optimization of reaction conditions for the $\mathrm{C}-\mathrm{H}$ functionalization of 3-Me indole with $\mathrm{Br}$-malonate

Changes from initial reaction
conditions

aReaction conditions: 0.4 mmol 3-Me indole (1a, 1 eq), Brmalonate ( 2 equiv), $\mathrm{Ph}_{3} \mathrm{~N}$ (1.5 equiv), $1.0 \mathrm{~mol} \%$ photocatalyst $\mathbf{D}$ in $2.5 \mathrm{ml} \mathrm{DMF}(0.16 \mathrm{M})$, irradiation with $465 \mathrm{~nm}$ LEDs, 5 hours reaction time. ${ }^{\text {bY } Y i e l d ~ d e t e r m i n e d ~ b y ~}$ GC-FID with decafluorobiphenyl as internal standard.

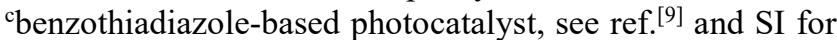
structure. ${ }^{\mathrm{d} I s o l a t e d ~ y i e l d . ~}{ }^{\mathrm{e}}$ For detailed flow conditions, see SI. 
The same transformation was previously reported to proceed with either $\mathrm{Ru}(\mathrm{bpy}) 3^{2+}$ or with an organic photocatalyst, and was therefore selected as benchmark reaction. ${ }^{[9,15]}$ As depicted in Table 1, we initially tested the ability of our photocatalysts to catalyse the reaction between 3-Me indole (1a) and Br-malonate in presence of triphenylamine as sacrificial electron donor. Subjected to blue LED irradiation, $87 \%$ of the desired alkylated indole (2a) was obtained within 5 hours with $1 \mathrm{~mol} \%$ of photocatalyst D (Table 1, entry 1). All other members of the series were tested under identical conditions and resulted in similar or inferior yields (see SI section 6). Gratifyingly, our photocatalyst outperformed Ru(bpy) $3^{2+}$ (Table $\mathbf{1}$ entry 3), and compared positively with the benzothiadiazole-based organic photocatalyst Th-BT-Th (Table 1, entry 2, reference ${ }^{[9]}$, see SI section 6 for structure). In order to better match the irradiation wavelength with the photocatalyst maximum absorption, we then conducted the reaction under irradiation with $400 \mathrm{~nm}$ LEDs, obtaining $75 \%$ of product within only 30 minutes. (Table 1, entry 4). Higher concentrations of photocatalyst did not result in increased amounts of product (Table 1 entry 5 and 6). Control experiments revealed that both the presence of light and of the photocatalyst were essential to achieve product formation (Table 1 entry 7, and 8). Finally, in order to overcome the limitations associated with photochemical reactions performed in batch reactors (i.e. limited light penetration, sub-optimal mixing), we implemented the use of a continuous flow capillary microreactor (I.D. $760 \mu \mathrm{m}, 2.5 \mathrm{~mL}$ reactor volume). ${ }^{[16]}$ Owing to the efficient irradiation of the reaction mixture achievable in microflow reactors, a good isolated yield of 2a (70\%) was obtained within only 7 minutes of residence time (Table 1, entry 9).

With optimized conditions in hand, we then explored the scope of this transformation (Scheme 1). A series of heteroaromatic compounds were tested: both N-protected and unprotected indoles underwent the reaction in good to excellent yields (2a-b). The functional group tolerance towards halides (2c) and esters (2d) was demonstrated.

The reaction proceeded with good yield also on a protected tryptophan moiety (2f), thus suggesting a possible application of this methodology in the context of bioconjugation chemistries. ${ }^{[17]} 3-\mathrm{Me}$ benzofuran was also successfully alkylated (2e). To further demonstrate the utility of this transformation, a series of 5-membered heterocycles were tested: pyrroles $(\mathbf{3 a}, \mathbf{3 b})$, thiophene $(\mathbf{3 c})$ and furans $(\mathbf{3 d}, \mathbf{3 e})$ were all found to be competent substrates, yielding the corresponding alkylated products in moderate to excellent yields.

Encouraged by the positive results obtained for the C$\mathrm{H}$ functionalization of heteroarenes with malonate derivatives, we turned our attention to the possibility of employing our organic photocatalysts for the $\mathrm{C}-\mathrm{H}$ trifluoromethylation and difluoroalkylation of heteroarenes. Due to the importance of fluorinated moieties in structure-activity relationship (SAR) studies, novel methodologies for the introduction of fluorine atoms in drug candidates are in high demand in the pharmaceutical industry. ${ }^{[18]}$
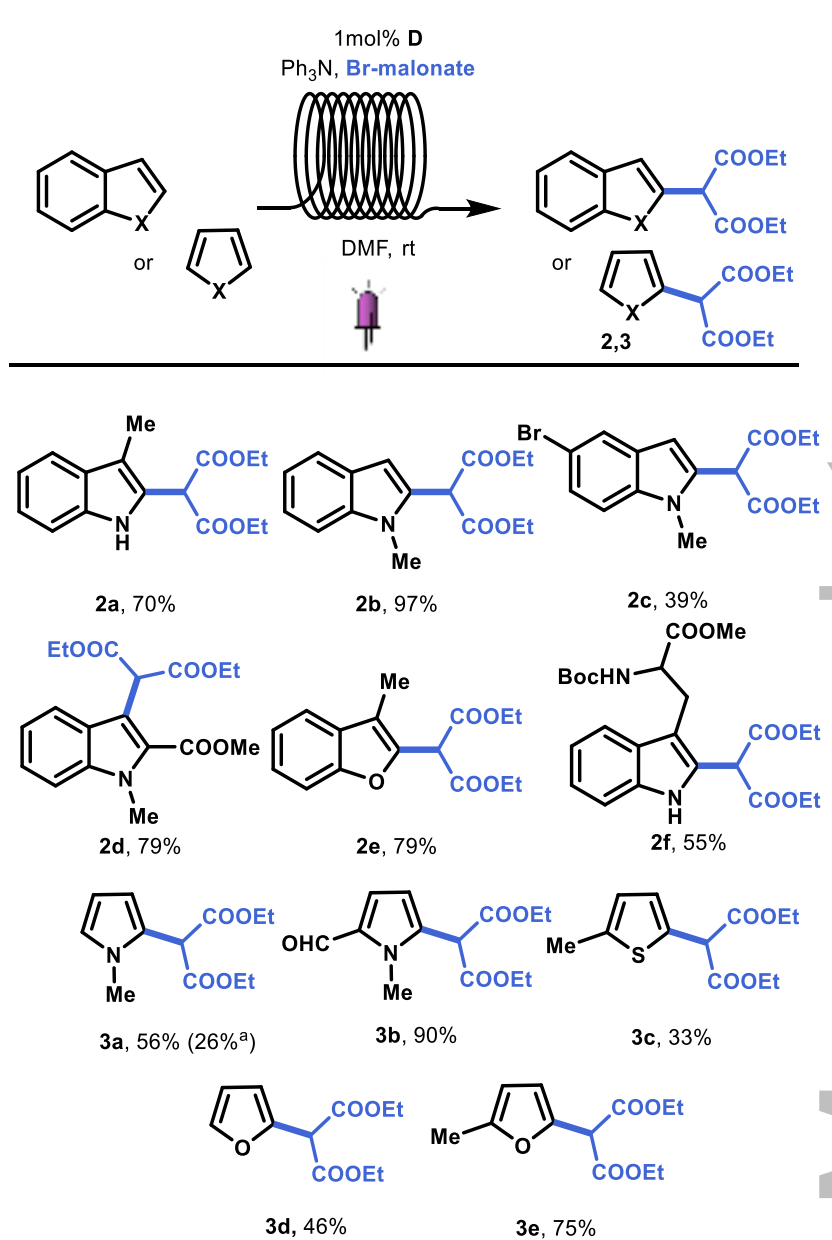

Scheme 1: Scope of the C-H functionalization of heteroarenes with malonate derivatives. Reaction conditions: $0.4 \mathrm{mmol}$ of substrate, $1 \mathrm{~mol} \% \mathbf{D}, 1.5$ equiv. of $\mathrm{Ph}_{3} \mathrm{~N}, 2$ equiv. Br-malonate in $2.5 \mathrm{ml}$ DMF $(0.16 \mathrm{M})$. Reactions performed in a capillary microflow reactor, irradiation with $400 \mathrm{~nm}$ LEDs, with different residence times depending on the substrate, isolated yields a Amount of di-substituted product obtained.

In this context, mild methodologies for the late-stage trifluoromethylation of potential drug candidates occupy a prominent role. Several photoredox-based trifluoromethylation methodologies have been reported in the last years, relying mostly on the use of expensive transition metal-based photoredox catalyst. ${ }^{[19]}$ We commenced our attempts toward the trifluoromethylation of heteroarenes in continuous flow by employing photocatalyst $\mathbf{D}$, gaseous and inexpensive trifluoromethyl iodide as trifluoromethylating agent and $N, N, N^{\prime}, N^{\prime}-$ Tetramethylethane-1,2-diamine (TMEDA) as sacrificial electron donor. ${ }^{[20]}$ 


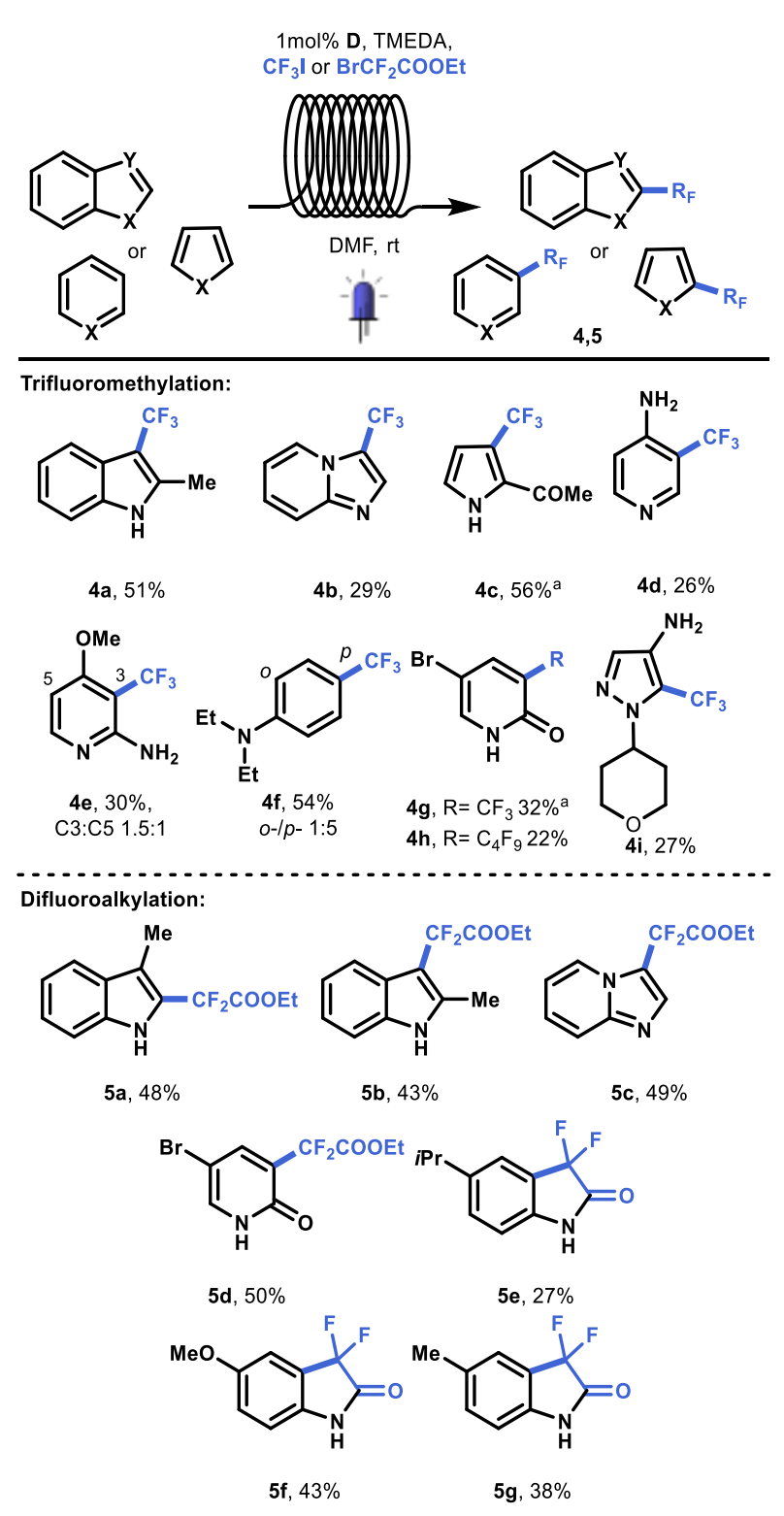

Scheme 2: Scope of the trifluoromethylation and difluoroalkylation of heteroarenes. Reaction conditions: 0.5 mmol of substrate, $1 \mathrm{~mol} \% \mathrm{D}, 3$ equiv. TMEDA, 4 equiv. $\mathrm{CF}_{3} \mathrm{I} / 2$ equiv. $\mathrm{BrCF}_{2} \mathrm{COOEt}$ in $5 \mathrm{ml}$ DMF (0.1M). Reactions performed in a Vapourtec UV-150 photoreactor, irradiation with $450 \mathrm{~nm}$ blue LEDs and $20 \mathrm{~min}$ residence

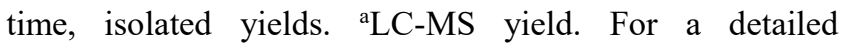
procedure for compounds $5 \mathrm{e}, 5 \mathrm{f}$ and $5 \mathrm{~g}$, we refer to SI, section 8 .

Notably, the formation of a gas-liquid slug flow regime in our continuous system resulted in improved mixing, while granting an efficient and homogeneous irradiation of the reaction mixture and affording a safe handling of the gas reactant. ${ }^{[16]}$ The results obtained for the trifluoromethylation of a diverse set of heteroarenes are summarized in Scheme 2. It is worth noting that, under analogous reaction conditions, a similar gas-liquid trifluoromethylation had been reported by our research group with either $\mathrm{Ru}(\mathrm{bpy})_{3}{ }^{2+}$ or with Eosin Y. ${ }^{[21]}$ Gratifyingly, the results obtained with photocatalyst D were comparable with those obtained with the other two photocatalyst. Moreover, a broader reaction scope (i.e. functionalization of complex 5- and 6-membered heterocycles) was demonstrated in this case.

Both trifluoromethylated indole (4a) and imidazo[1,2a]pyridine (4b) derivatives were obtained in synthetically useful yields, while the trifluoromethylated pyrrole derivative 4c was obtained with a 56\% yield. Pyridine derivatives 4d and $\mathbf{4 e}$ were isolated in modest yields. Unfortunately, for compounds obtained in modest yields prolonging reaction time in the photoreactor did not contribute to increase the conversion of starting materials.

Next, the applicability of our methodology to other nitrogen containing heterocycles was demonstrated with compounds $\mathbf{4 g}$ and $4 \mathbf{i}$. Notably, the perfluoroalkylated derivative $\mathbf{4 h}$, obtained via the use of $\mathrm{IC}_{4} \mathrm{~F}_{9}$ as fluorinating agent, was also isolated in a $22 \%$ yield.

Finally, the aniline derivative 4 f was obtained in $54 \%$ yields as a separable mixture of ortho- and pararegioisomers. In order to further demonstrate the reactivity of the develop photocatalysts, we then focused on the introduction of a difluoroester moiety (Fehler! Verweisquelle konnte nicht gefunden werden.), which is a precursor of high interest in the preparation of radio-labeled ${ }^{18} \mathrm{~F}$ compounds for positron-emission tomography (PET) scan. ${ }^{[22],[23]}$ By employing $\mathrm{Br}-\mathrm{CF}_{2} \mathrm{COOEt}$ as fluorinating agent, the corresponding indoles $\mathbf{( 5 a}, \mathbf{5 b})$ and imidazo[1,2a]pyridine (5c) derivatives were obtained in good yields. Similarly, the Br-pyridone derivative 5d was obtained in $50 \%$ yield. Interestingly, when aniline derivatives were employed for this transformation a one-pot tandem difluoroalkylation/amidation was observed, resulting in the formation $\mathrm{o}_{1}$ difluorooxindoles derivatives 5e-g. ${ }^{[24]}$

Fluorescence quenching studies on the reactions studied demonstrated that both reductive and oxidative single electron transfers (SET) are viable pathways for the excited state of photocatalyst D (see SI section 7). Such versatility in terms of redox properties further highlights the potential of this novel photocatalyst series in a broad range of photocatalytic reactions.

In conclusion, we reported the rational de novo synthesis of a novel class of 2,2'-bithiophene-based organic photocatalysts. DFT calculations were used to predict a priori the spectroscopic properties and the redox potentials of an array of molecules, of which six were then synthesized and characterized. The experimental data were found to be in good agreement with the theoretical values. The activity of this class of molecules as photocatalyst was then showcased in the $\mathrm{C}-\mathrm{H}$ functionalization of heteroarenes with malonate derivatives. The activity of the tested compounds was comparable with that of other commonly used organic or transition-metal photocatalysts (i.e. Th-BT-Th and $\mathrm{Ru}(\mathrm{bpy}){ }_{3}{ }^{2+}$ ), and a series of derivatives was obtained in good to excellent yields. Process intensification in a microflow photoreactor afforded increased yields and reduced reaction times. Moreover, the implementation of our photocatalyst for the trifluoromethylation and 
difluoroalkylation of heteroarenes and benzofused heteroarenes was also demonstrated. Mechanistic insight via fluorescence quenching studies and SternVolmer analysis indicated that these photocatalyst can undergo both reductive and oxidative quenching pathways. Due to their inexpensive nature, good absorption in the $400 \mathrm{~nm}$ range and their favourable redox potentials, we believe that this novel class of 2,2'-bithiophene-based organic photocatalyst could find interesting applications in photoredox catalysis.

\section{Experimental Section}

\section{General procedure for the synthesis of photocatalysts A-F}

A mixture of 5,5'-dibromo-2,2'-bithiophene $(0.100 \mathrm{~g}, 0.31$ $\mathrm{mmol})$, the corresponding acetylene derivative $(0.77 \mathrm{mmol})$, DBU $(0.094 \mathrm{~g}, 0.62 \mathrm{mmol}), \mathrm{CuI}(0.0017 \mathrm{~g}, 0.015 \mathrm{mmol})$ and Pd-Encat ${ }^{\mathrm{TM}}$ TPP30 (Palladium acetate and triphenylphosphine, microencapsulated in a polyurea matrix, $0.026 \mathrm{~g}, 0.035 \mathrm{mmol}$ ) was charged under argon to a dried microwave vessel. MeCN (1 mL) was added. The vessel was closed and irradiated at $140{ }^{\circ} \mathrm{C}$ for $25 \mathrm{~min}$. The crude reaction product was purified by chromatography, eluting with hexane/ethyl acetate to give analytically pure photocatalysts A to $\mathbf{F}$.

General procedure for the $\mathrm{C}-\mathrm{H}$ functionalization of heteroarenes with malonate derivatives in continuous flow

In an oven-dried vial equipped with a magnetic stirrer and a PTFE septum, $2.0 \mathrm{mg}(1 \mathrm{~mol} \mathrm{\%})$ of RA48 was added to a mixture of the hetero-arene substrate $(0.4 \mathrm{mmol}, 1$ equiv. $)$, Br-malonate $(0.8 \mathrm{mmol}, 2$ equiv.) in $1.25 \mathrm{ml}$ of DMF. In another vial, $\mathrm{Ph}_{3} \mathrm{~N}(0.6 \mathrm{mmol}, 1.5$ equiv) was dissolved in $1.25 \mathrm{ml}$ of DMF. The two solution were merged with a Tmixer prior entering the microcapillary flow reactor (PFA tubing, $0.76 \mathrm{~mm}$ ID, $2.5 \mathrm{~mL}$ reactor volume). The reactor was irradiated with $400 \mathrm{~nm}$ purple LEDs. For the residence time of every substrate we refer to the compound characterization in SI. The reaction mixture collected from the outlet was diluted with $\mathrm{H}_{2} \mathrm{O}$ and extracted with $\mathrm{Et}_{2} \mathrm{O}$ $(2 \mathrm{x})$ and EtOAc $(1 \mathrm{x})$. The combined organic layers were washed with brine, dried over $\mathrm{MgSO}_{4}$ and concentrated in vacuo. The crude was then pre-adsorbed onto silica, dried in vacuo and purified by flash chromatography to yield the desired alkylated product.

\section{General procedure for the trifluoromethylation and difluoroalkylation of heteroarenes in continuous flow}

In an oven-dried vial equipped with a magnetic stirrer and a PTFE septum, $2.5 \mathrm{mg}(1 \mathrm{~mol} \%)$ of RA48 was added to a mixture of the hetero-arene substrate $(0.5 \mathrm{mmol}, 1$ equiv. $)$, $\mathrm{BrCF}_{2} \mathrm{COOEt}$ (1.0 mmol, 2 equiv.) in $2.5 \mathrm{ml}$ of DMF. In another vial, TMEDA (1.5 mmol, 3 equiv) was dissolved in $2.5 \mathrm{ml}$ of DMF. The two solution were merged with a Tmixer prior entering the Vapourtec Photoreactor (fluoropolymer tube, $1.3 \mathrm{~mm}$ ID, $5 \mathrm{~mL}$ ) and the total liquid flowrate was set to $0.25 \mathrm{~mL} / \mathrm{min}$ (20 minutes residence time). For trifluoromethylation reaction, gaseous $\mathrm{CF}_{3} \mathrm{I}$ was added via a mass flow controller $(2.45 \mathrm{ml} / \mathrm{min}, 4 \mathrm{eq})$ and the gaseous stream was merged with the liquid one with a Tmixer prior to entering the photoreactor. The reactor was irradiated with 54 blue LEDs $(450 \mathrm{~nm}$, total power $24 \mathrm{~W})$. The reaction mixture collected from the outlet was diluted with $\mathrm{H}_{2} \mathrm{O}$ and extracted with $\mathrm{Et}_{2} \mathrm{O}(2 \mathrm{x})$ and EtOAc (1x). The combined organic layers were washed with brine, dried over $\mathrm{MgSO}_{4}$ and concentrated in vacuo. The crude was then pre-adsorbed onto silica, dried in vacuo and purified by flash chromatography to yield the desired alkylated product.

\section{Acknowledgements}

C.B., I.A., J.A. and T.N. acknowledge the European Union for a Marie Curie ITN Grant (Photo4Future, Grant No. 641861). Financial support from Junta de Comunidades de Castilla La Mancha (JCCM-FEDER) (project SBPLY/17/180501/000189) and Gobierno de Aragón, grupo de referencia E14_17R is acknowledged. R. Martín is grateful to MEC for an FPU studentship. Moreover, technical support from the High Performance Computing Service of the University of Castilla La Mancha is gratefully acknowledged. The authors would like to acknowledge Mr. Alberto Fontana for his help with LC-MS analysis.

\section{References}

[1] a) C. K. Prier, D. A. Rankic, D. W. C. MacMillan, Chem. Rev. 2013, 113, 5322-5363; b) J. W. Tucker, Y. Zhang, T. F. Jamison, C. R. J. Stephenson, Angew. Chem., Int. Ed. 2012, 51, 4144-4147.

[2] a) K. L. Skubi, T. R. Blum, T. P. Yoon, Chem. Rev. 2016, 116, 10035-10074; b) J. M. R. Narayanam, C. R. J. Stephenson, Chem. Soc. Rev. 2011, 40, 102-113.

[3] D. M. Arias-Rotondo, J. K. McCusker, Chem. Soc. Rev. 2016, 45, 5803-5820.

[4] N. A. Romero, D. A. Nicewicz, Chem. Rev. 2016, 116, 10075-10166.

[5] a) D. A. Nicewicz, T. M. Nguyen, ACS Catal. 2014, 4 355-360; b) A. Joshi-Pangu, F. Lévesque, H. G. Roth, S F. Oliver, L.-C. Campeau, D. Nicewicz, D. A. DiRocco, J. Org. Chem. 2016, 81, 7244-7249; c) E. Speckmeier, T. Fischer, K. Zeitler, J. Am. Chem. Soc. 2018, DOI: 10.1021/jacs.8b08933.

[6] a) M. Majek, F. Filace, A. J. v. Wangelin, Beilstein J. Org. Chem. 2014, 10, 981-989; b) G. Oster, N. Wotherspoon, J. Chem. Phys. 1954, 22, 157-158.

[7] a) J. Luo, J. Zhang, ACS Catal. 2016, 6, 873-877; b) H. Uoyama, K. Goushi, K. Shizu, H. Nomura, C. Adachi, Nature 2012, 492, 234-238.

[8] a) W.-I. Hung, Y.-Y. Liao, C.-Y. Hsu, H.-H. Chou, T.H. Lee, W.-S. Kao, J. T. Lin, Chem. - Asian J. 2014, 9, 357-366; b) Y. Lin, Y. Li, X. Zhan, Chem. Soc. Rev. 2012, 41, 4245-4272; c) K. Takimiya, M. Nakano, Bull. Chem. Soc. Jpn. 2018, 91, 121-140.

[9] L. Wang, W. Huang, R. Li, D. Gehrig, P. W. M. Blom, K. Landfester, K. A. I. Zhang, Angew. Chem., Int. Ed. 2016, 55, 9783-9787.

[10] a) R. G. Parr, W. Yang, in Density-Functional Theory of Atoms and Molecules, Oxford University Press, USA, 1994; b) C. Adamo, D. Jacquemin, Chem. Soc. Rev. 2013, 42, 845-856.

[11] Y. Zhao, D. G. Truhlar, Theor. Chem. Acc. 2007, 120, 215-241.

[12] a) J. Andzelm, C. Kölmel, A. Klamt, J. Chem. Phys. 1995, 103, 9312-9320; b) M. Cossi, N. Rega, G. Scalmani, V. Barone, J. Comput. Chem. 2003, 24, 669681. 
[13] a) R. Martín, P. Prieto, J. R. Carrillo, I. Torres, C. A. Strassert, K. Soloviova, A. M. Rodríguez, L. Sánchez, Á. Díaz-Ortiz, Dyes Pigm. 2018, 151, 327-334; b) M. J. Pastor, I. Torres, C. Cebrián, J. R. Carrillo, Á. DíazOrtiz, E. Matesanz, J. Buendía, F. García, J. Barberá, P. Prieto, L. Sánchez, Chem. - Eur. J. 2015, 21, 1795-1802.

[14] N. Elgrishi, K. J. Rountree, B. D. McCarthy, E. S. Rountree, T. T. Eisenhart, J. L. Dempsey, J. Chem. Educ. 2018, 95, 197-206.

[15] L. Furst, B. S. Matsuura, J. M. R. Narayanam, J. W. Tucker, C. R. J. Stephenson, Org. Lett. 2010, 12, 31043107.

[16] a) D. Cambié, C. Bottecchia, N. J. W. Straathof, V. Hessel, T. Noël, Chem. Rev. 2016, 116, 10276-10341; b) M. B. Plutschack, B. Pieber, K. Gilmore, P. H. Seeberger, Chem. Rev. 2017, 117, 11796-11893.

[17] C. Bottecchia, T. Noel, Chem. - Eur. J. 2018, DOI: 10.1002/chem.201803074.

[18] a) S. Purser, P. R. Moore, S. Swallow, V. Gouverneur, Chem. Soc. Rev. 2008, 37, 320-330; b) Y. Zhou, J. Wang, Z. Gu, S. Wang, W. Zhu, J. L. Aceña, V. A. Soloshonok, K. Izawa, H. Liu, Chem. Rev. 2016, 116, 422-518.

[19] a) I. Abdiaj, C. Bottecchia, J. Alcazar, T. Noël, Synthesis 2017, 49, 4978-4985; b) T. Chatterjee, N. Iqbal, Y. You, E. J. Cho, Acc. Chem. Res. 2016, 49, 2284-2294; c) J. W. Beatty, J. J. Douglas, K. P. Cole, C. R. J. Stephenson, Nat. Commun. 2015, 6, 7919; d) N. Iqbal, J. Jung, S. Park, E. J. Cho, Angew. Chem., Int. Ed. 2014, 53, 539-542; e) D. A. Nagib, D. W. C. MacMillan, Nature 2011, 480, 224-228; f) N. Iqbal, S. Choi, E. Kim, E. J. Cho, J. Org. Chem. 2012, 77, 11383-11387.

[20] N. Iqbal, S. Choi, E. Ko, E. J. Cho, Tetrahedron Lett. 2012, 53, 2005-2008.

[21] a) N. J. W. Straathof, H. P. L. Gemoets, X. Wang, J. C. Schouten, V. Hessel, T. Noël, ChemSusChem 2014, 7, 1612-1617; b) N. Straathof, D. Osch, A. Schouten, X. Wang, J. Schouten, V. Hessel, T. Noël, J. Flow Chem. 2015, 4, 12-17.

[22] a) T. Khotavivattana, S. Verhoog, M. Tredwell, L. Pfeifer, S. Calderwood, K. Wheelhouse, T. Lee Collier, V. Gouverneur, Angew. Chem., Int. Ed. 2015, 54, 99919995; b) S. Preshlock, M. Tredwell, V. Gouverneur, Chem. Rev. 2016, 116, 719-766; c) P. W. Miller, N. J. Long, R. Vilar, A. D. Gee, Angew. Chem., Int. Ed. 2008, 47, 8998-9033.

[23] a) J. Jung, E. Kim, Y. You, E. J. Cho, Adv. Synth. Catal. 2014, 356, 2741-2748; b) X. Pan, H. Xia, J. Wu, Org. Chem. Front. 2016, 3, 1163-1185.

[24] L.-C. Yu, J.-W. Gu, S. Zhang, X. Zhang, J. Org. Chem. 2017, 82, 3943-3949. 


\section{COMMUNICATION}

De novo design of organic photocatalysts:

bithiophene derivatives for the visible-light induced $\mathrm{C}-\mathrm{H}$ functionalization of heteroarenes

Adv. Synth. Catal. Year, Volume, Page - Page

Cecilia Bottecchia ${ }^{a \dagger}$, Raúl Martín ${ }^{\mathrm{b} \dagger}$, Irini Abdiaj ${ }^{\mathrm{c}}$, Ettore Crovini ${ }^{\mathrm{a}}$, Jesús Alcazar ${ }^{\mathrm{c}}$, Jesús Orduna ${ }^{\mathrm{d}}$,

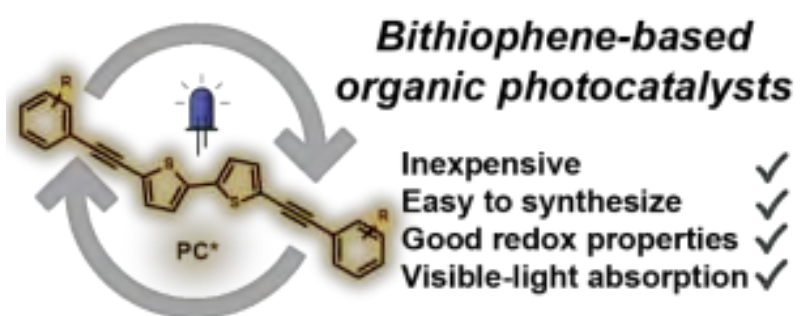
María Jesús Blesa ${ }^{\mathrm{d}}$, José R. Carrillo ${ }^{\mathrm{b} *}$, Pilar Prieto ${ }^{\mathrm{b}}$ and Timothy Noël ${ }^{\text {a* }}$ 